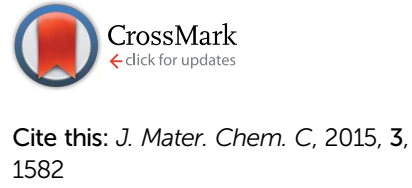

Received 21st November 2014 Accepted 17th December 2014

DOI: $10.1039 / \mathrm{c} 4 \mathrm{tc} 02664 \mathrm{k}$

www.rsc.org/MaterialsC

\section{Microporous, tetraarylethylene-based polymer networks generated in a reductive polyolefination process $\uparrow$}

\begin{abstract}
E. Preis, ${ }^{a}$ W. Dong, ${ }^{a}$ G. Brunklaus ${ }^{b}$ and U. Scherf ${ }^{\star a}$
Microporous Polymer Networks (MPNs) that contain aggregate-induced emission (AIE) active tetraphenylethylene (TPE) or other tetraarylethylene units have been generated in a reductive polyolefination process starting from four different tris $(\alpha, \alpha$-dichlorobenzyl)arene derivatives with dicobalt octacarbonyl or chromium(॥)acetate as reductive olefination agents. Microporosity with moderately high BET surface areas up to $500 \mathrm{~m}^{2} \mathrm{~g}^{-1}$ could be combined with high solid state photoluminescence quantum yields up to $25.3 \%$ for polymer network P4. This unique combination should be promising for applications as optical solid state sensors, especially for MPNs with electron-deficient triazine core units.
\end{abstract}

\section{Introduction}

Microporous Polymer Networks (MPNs) comprise a rapidly growing area of materials science towards future mass applications in catalysis, gas storage/separation or as active porous materials for organic electronic devices and sensors. ${ }^{1-7}$ For such mass applications inexpensive synthesis procedures for MPN generation without use of noble metal catalysts and under mild reaction conditions are of particular appeal. ${ }^{8-18}$ Tetraphenylethylene (TPE) and related repeat units represent versatile building blocks for the rational design of MPNs due to their rigid, three-dimensional structure and interesting optical properties, especially a distinct aggregate-induced emission (AIE). Linear poly-TPEs (as representative example poly(1,4phenylene-1,2-diphenylvinylene) DP-PPV) was first described 30 years ago by Hörhold and co-workers. ${ }^{19}$ In the following decades, a rather broad variety of TPE-based monomers, oligomers and (co)polymers have been extensively investigated by Tang and co-workers thereby documenting their broad and very promising application potential in materials science and biology. ${ }^{20-24}$ The first synthesis of MPNs that contain TPE building blocks and show a pronounced AIE effect has been reported by Jiang and co-workers. ${ }^{25,26}$ Their MPNs have been generated in a Yamamoto-type aryl-aryl coupling starting from tetrabromo-TPE with $\mathrm{Ni}(\mathrm{COD})_{2}$ as coupling reagent. In this study, we now introduce the synthesis of structurally related

\footnotetext{
${ }^{a}$ Bergische Universität Wuppertal, Macromolecular Chemistry Group (buwmakro) and Institute for Polymer Technology, Gauss-Str. 20, D-42119 Wuppertal, Germany. E-mail: scherf@uni-wuppertal.de

${ }^{b}$ Westfälische Wilhelms-Universität, Institut für Physikalische Chemie, Corrensstr. 28, D-48149 Münster, Germany

$\dagger$ Electronic supplementary information (ESI) available. See DOI: $10.1039 / \mathrm{c} 4 \mathrm{tc} 02664 \mathrm{k}$
}

poly-TPE networks in a reductive olefination process starting from tris $(\alpha, \alpha$-dichlorobenzyl)arene monomers with dicobalt octacarbonyl $^{27,28}$ or chromium(II)acetate ${ }^{19,29}$ as condensing agents. In our target networks the tetraarylethylene units (Scheme 1) are linked together via 1,3,5-substituted benzene or 2,4,6-substituted 1,3,5-triazine cores. Jiang's MPNs, in contrast, contain TPE units with the olefinic double bonds connected via biphenyl units. ${ }^{25}$

The synthesis of the monomers M1-M4 is depicted in Scheme 2 (for detailed information see ESI $\dagger$ ). The general synthesis route of the polymer networks P1-P4 is outlined in Scheme 3 for P1 as representative example. 1,3,5-Tribenzoylbenzene was prepared according to a reported procedure. $^{30}$ The until now unknown, corresponding 1,3,5$\operatorname{tris}(\alpha, \alpha$-dichlorobenzyl)benzene MCl1 was generated by reaction with phosphorus pentachloride $\left(\mathrm{PCl}_{5}\right)$ in chlorobenzene as solvent (Scheme 3). After removal of both phosphorus oxychloride $\left(\mathrm{POCl}_{3}\right)$ by distillation and excess $\mathrm{PCl}_{5}$ by sublimation, respectively, the resulting oily product was used without further purification, similar to the synthesis of bifunctional analogues as 1,3 -bis $(\alpha, \alpha$-dichlorobenzyl)benzene (made for polyolefination into linear coupling products). ${ }^{31} \mathrm{H}$ NMR spectroscopy indicated the presence of pure $1,3,5-\operatorname{tris}(\alpha, \alpha-$ dichlorobenzyl)benzene MCl1 (Fig. 1). The singlet of the 1,3,5substituted benzene core is, thereby, shifted from $8.40 \mathrm{ppm}$ to 7.83 ppm.

For the synthesis of monomer M2, 1,3,5-tris(4-bromophenyl) benzene was prepared following a literature procedure. ${ }^{32}$ Subsequent nucleophilic substitution with copper(I) cyanide yielded 1,3,5-tris(4-cyanophenyl)benzene (Scheme 2); the rather low yield is related to an ongoing trimerization of nitrile groups into triazine-cored byproducts. ${ }^{33}$ Addition of phenylmagnesium bromide, followed by hydrolysis with diluted hydrochloric acid afforded 1,3,5-tris[4-benzoylphenyl]benzene. The corresponding 


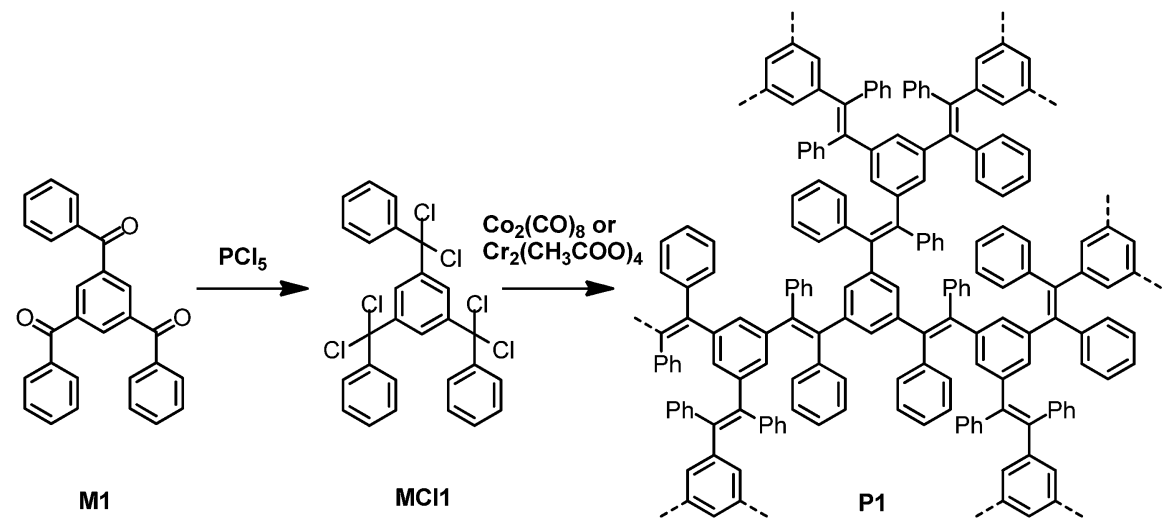

Scheme 3 Synthesis of the TPE-based MPN P1 in a reductive polyolefination.

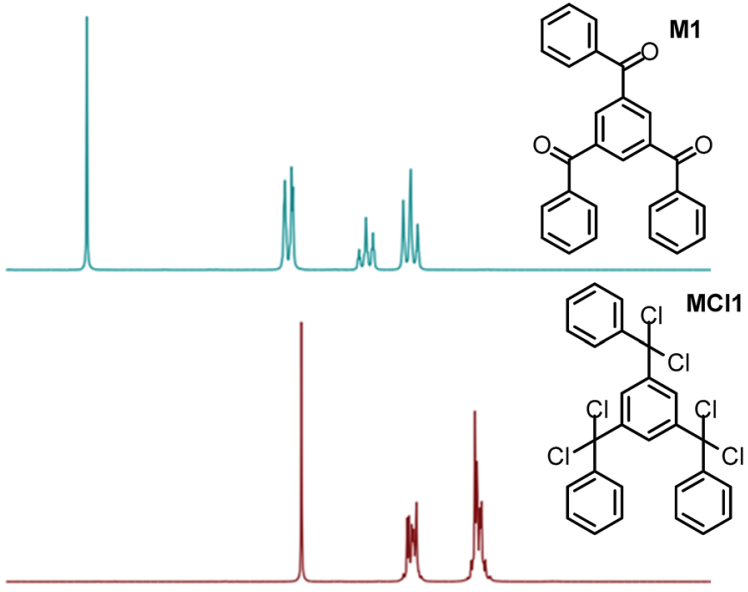

$\begin{array}{llllllllllllllllllllllll}8.6 & 8.5 & 8.4 & 8.3 & 8.2 & 8.1 & 8.0 & 7.9 & 7.8 & 7.7 & 7.6 & 7.5 & 7.4 & 7.3 & 7.2 & 7.1 & 7.0 & 6.9 & 6.8\end{array}$

Fig. $1{ }^{1} \mathrm{H}$ NMR spectra of: (A) 1,3,5-tribenzoylbenzene $\mathrm{M} 1$ and (B) 1,3,5-tris $\left(\alpha, \alpha\right.$-dichlorobenzyl)benzene $\mathrm{MCl}$ in $\mathrm{C}_{2} \mathrm{D}_{2} \mathrm{Cl}_{4}$.

1,3,5-tris[4-( $\alpha, \alpha$-dichlorobenzyl)phenyl]benzene $\mathrm{MCl} 2$ intermediate was then generated as described for $1,3,5$-tris $(\alpha, \alpha$-dichlorobenzyl)-benzene, also resulting in an oily product. Furthermore, the synthesis of tris(2,4,6-benzoyl)-1,3,5-triazine M3 was accomplished following an already published approach. ${ }^{34-36}$ Its subsequent transformation into 2,4,6-tris( $\alpha, \alpha$-dichlorobenzyl)-1,3,5triazine $\mathrm{MCl} 3$ was carried out as described for MCl1 and MCl2. The ${ }^{1} \mathrm{H}$ NMR spectrum of the corresponding hexachloro-derivative $\mathrm{MCl} 3$ exhibits two aromatic signals, a doublet at $7.62 \mathrm{ppm}$ $(6 \mathrm{H})$ and a multiplet at 7.36-7.30 $(9 \mathrm{H})$ thus demonstrating a complete transformation of carbonyl into $\alpha, \alpha$-dichloromethyl groups. The preparation of 2,4,6-tris(4-methylphenyl)-1,3,5triazine and its subsequent oxidation to the corresponding tricarboxylic acid (Scheme 2) was achieved by applying literature methods. ${ }^{37,38}$ The reaction of the corresponding acid chloride with benzene after Friedel-Crafts resulted in monomer M4, 2,4,6tris(4-benzoylphenyl)-1,3,5-triazine, that was finally converted into the corresponding 2,4,6-tris[4-( $\alpha, \alpha$-dichlorobenzyl)phenyl]-1,3,5triazine MCl4 as outlined for MCl1-MCl3. In the ${ }^{13} \mathrm{C}$ NMR spectrum of the hexachloro-substituted monomer $\mathrm{MCl} 4$ complete disappearance of the carbonyl signal at $196.6 \mathrm{ppm}$ and formation of an aliphatic signal at $91.7 \mathrm{ppm}\left(\mathrm{ar}_{2} \mathrm{CCl}_{2}\right)$ confirmed the complete conversion.

The resulting, moisture sensitive hexachloro monomers prepared from the tribenzoyl derivatives M1-M4 were used without further purification, as already mentioned. Their reductive polyolefination into the polymer networks P1-P4 was accomplished by using two different reducing/condensing agents: (i) dicobalt octacarbonyl $\mathrm{Co}_{2}(\mathrm{CO})_{8}$ (ref. 27 and 28) or (ii) chromium(II)acetate $\mathrm{Cr}_{2} \mathrm{ac}_{4},{ }^{19,29}$ respectively. The insoluble coupling products precipitated during reaction and were isolated as yellowish colored solids. The polymeric products P1, P2 and P4 show yellow photoluminescence (PL) of different intensity (P3: no PL detected, see Table 2) and were subsequently extracted with several organic solvents (methanol, ethyl acetate, 1,4-dioxane, chloroform) and finally washed with supercritical carbon dioxide $\mathrm{sc}-\mathrm{CO}_{2}{ }^{16,18}$ prior to further characterization.

The absence of carbonyl signals at 185-200 ppm (generated by hydrolysis of $-\mathrm{CCl}_{2}$ - functions) in the solid-state ${ }^{13} \mathrm{C}$ CPMAS spectra of the polymer networks P1-P4 (all made with $\mathrm{Co}_{2}(\mathrm{CO})_{8}$ as reducing agent, for P4 see Fig. 2, for P1-P3 see Fig. S5a-c, ESI $†$ ) clearly evidence a successful reductive polycondensation. In case of polymer networks that contain triazine cores (P3 and $\mathrm{P} 4$ ), an additional signal at about $170 \mathrm{ppm}$ is observed (as for

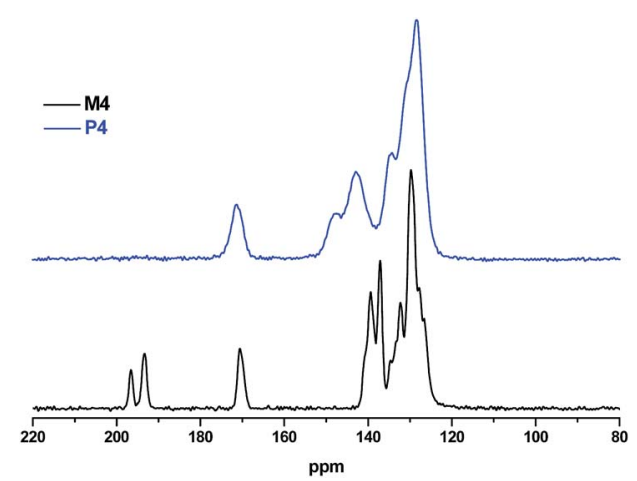

Fig. 2 Solid state ${ }^{13} \mathrm{C}$ NMR spectra of monomer M4 and polymer network P4. 


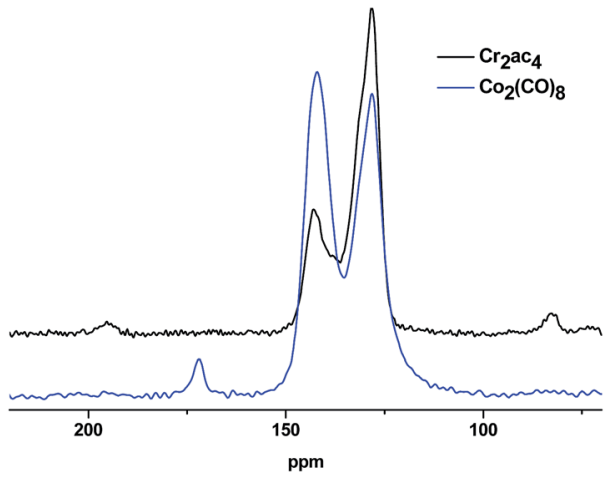

Fig. 3 Solid state ${ }^{13} \mathrm{C}$ NMR spectra of polymer network P1 made with $\mathrm{CO}_{2}(\mathrm{CO})_{8}$ (blue) or $\mathrm{Cr}_{2} \mathrm{ac}_{4}$ (black) as reducing agent.

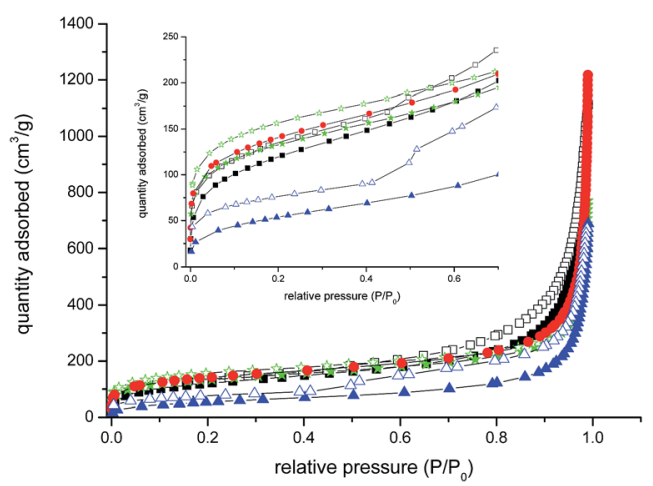

Fig. 4 Nitrogen adsorption (filled symbols)/desorption (open symbols) isotherms at $77 \mathrm{~K}$ for P1 (black squares), P2 (red circles), P3 (green stars), P4 (blue triangles); the inset shows the pressure range $P / P_{0}$ from 0 to 0.7 .

the monomers) that is characteristic for the 1,3,5-triazine unit. The rather weak signal at $170 \mathrm{ppm}$ for the triazine-free networks P1 and P2 may be related to carboxylic acid or ester functions that are formed in a side reaction, probably by $\mathrm{CO}$ insertion. In contrast to a P1 sample made with $\mathrm{Co}_{2}(\mathrm{CO})_{8}$ (Fig. 3), the ${ }^{13} \mathrm{C}$
CPMAS spectrum of another P1 sample obtained with $\mathrm{Cr}_{2} \mathrm{ac}_{4}$ as condensing agent exhibits two additional carbon signals at 195 ppm for leftover carbonyl groups and an aliphatic signal at 83 $\mathrm{ppm}$, but, interestingly, no signal around $170 \mathrm{ppm}$. Thereby, the presence of leftover carbonyl groups suggests incomplete conversion. For this reason only samples made with $\mathrm{Co}_{2}(\mathrm{CO})_{8}$ have been considered for further characterization.

The porosity of the polymer networks P1-P4 was studied by nitrogen gas adsorption and desorption at $77 \mathrm{~K}$. The networks P1-P4 all show type I isotherms (see Fig. 4) with a significant amount of nitrogen adsorbed at low pressure, followed by a much increased adsorption at higher pressure $\left(P / P_{0}>0.9\right)$ attributed to gas condensation into voids between polymer particles. ${ }^{39}$

As often observed for "soft" polymers networks, the desorption-branch did not connect to the adsorption-branch at low pressure. ${ }^{40}$ P3 additionally shows a distinct hysteresis, most probably due to capillary condensation (see Fig. 4). Moderately high BET surface areas of $462 \mathrm{~m}^{2} \mathrm{~g}^{-1}(\mathrm{P} 1), 502 \mathrm{~m}^{2} \mathrm{~g}^{-1}$ (P2), 200 $\mathrm{m}^{2} \mathrm{~g}^{-1}$ (P3), and $475 \mathrm{~m}^{2} \mathrm{~g}^{-1}$ (P4) could be determined for the relative pressure range of $0.01-0.15 P / P_{0}$ (see Table 1), while rather high total pore volumes of $1.05-1.87 \mathrm{~cm}^{3} \mathrm{~g}^{-1}$ have been found for P1-P4.

Carbon dioxide and methane uptakes of the networks were determined at $298 \mathrm{~K}$, the hydrogen uptake at $77 \mathrm{~K}$ up to a pressure of 1 bar. The gas uptake is significantly increased for the networks with additional phenylene "spacers" (P2 and P4) for all three gases, but most pronounced for $\mathrm{CH}_{4}$ as the largest guest molecule, thus indicating an increase of the pore diameter. The calculated selectivities for $\mathrm{CO}_{2} / \mathrm{CH}_{4}$ at $298 \mathrm{~K}$ are 4.7 (P1), $3.6(\mathrm{P} 2), 4.6(\mathrm{P} 3)$ and $3.6(\mathrm{P} 4)$, for $\mathrm{N}_{2} / \mathrm{H}_{2}$ at $77 \mathrm{~K}$ are 24.2 (P1), 19.3 (P2), 18.0 P3 and 12.6 (P4) (for details see the ESI $\dagger$ ).

Powder X-ray diffraction (PXRD) analysis of polymer network P4 (Fig. S26, ESI†) only displays three broad peaks of low intensity in the wide-angle region, thus suggesting a dominantly amorphous network structure. The peaks correspond to distances of $6.45,4.5$ and $2.1 \AA$, respectively, most probably

Table 1 Gas sorption properties of polymer networks P1-P4

\begin{tabular}{llllllll}
\hline & $\begin{array}{l}\text { BET surface area } \\
\left(\mathrm{m}^{2} \mathrm{~g}^{-1}\right)\end{array}$ & $\begin{array}{l}\text { Pore volume } \\
(\mathrm{cc})\end{array}$ & $\begin{array}{l}\mathrm{H} \text { uptake } \\
(\%)\end{array}$ & $\begin{array}{l}\mathrm{CO}_{2} \text { uptake } \\
(\%)\end{array}$ & $\begin{array}{l}\mathrm{CH}_{4} \text { uptake } \\
(\%)\end{array}$ & $\begin{array}{l}\text { Selectivity }\left(\mathrm{CO}_{2} / \mathrm{CH}_{4}\right) \\
(298 \mathrm{~K})\end{array}$ & $\begin{array}{l}\mathrm{Selectivity}\left(\mathrm{N}_{2} / \mathrm{H}_{2}\right) \\
(77 \mathrm{~K})\end{array}$ \\
\hline P1 & 462 & 1.76 & 0.44 & 1.46 & 0.11 & 4.7 & 24.2 \\
P2 & 502 & 1.87 & 0.57 & 2.54 & 0.26 & 3.6 & 19.3 \\
P3 & 200 & 1.05 & 0.35 & 1.58 & 0.12 & 4.6 & 18.0 \\
P4 & 475 & 1.16 & 0.55 & 2.58 & 0.26 & 3.6 & 12.6
\end{tabular}

Table 2 Optical and thermogravimetric (TGA) data of P1-P4

\begin{tabular}{|c|c|c|c|c|}
\hline P1 & 352 & 540 & 3.5 & $272(2.5 \%)$ \\
\hline P3 & 367 & no PL detected & - & $296(7.9 \%)$ \\
\hline P4 & 392 & 560 & 25.3 & $408(1.5 \%)$ \\
\hline
\end{tabular}




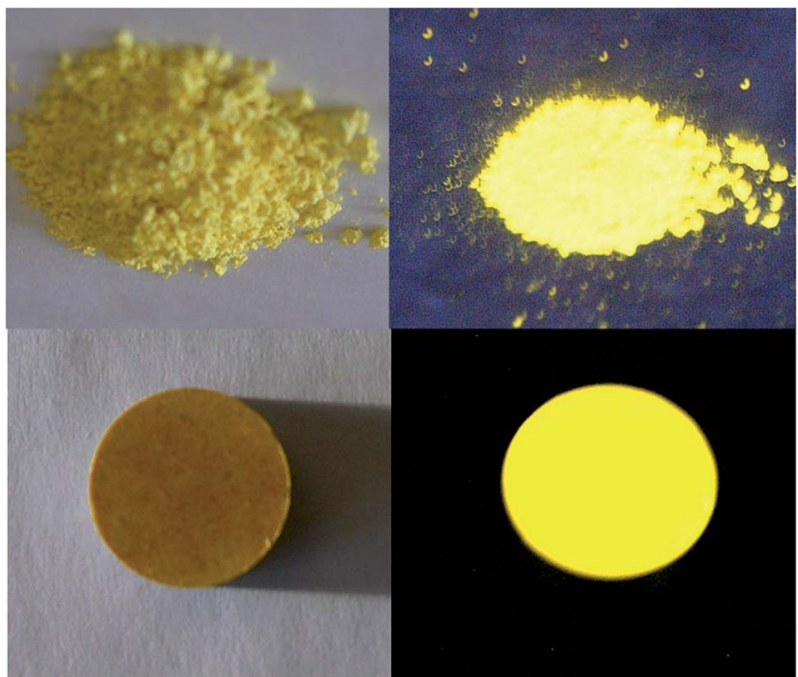

Fig. 5 P4 powder (top) and P4 pellet (down) at day light (left) and under UV irradiation (right).

reflecting periodic structural motifs of P4 (as TPE-TPE and triazine-triazine distances).

The solid state optical spectra of the polymer networks are depicted in Fig. 6. From the onset of the absorption features (Fig. S4, ESI $\dagger$ ) HOMO-LUMO energy gaps of 2.90 and $2.78 \mathrm{eV}$ are estimated for the polymer networks $\mathrm{P} 2$ and $\mathrm{P} 4$, respectively. The photoluminescence (PL) spectra of the polymer networks P1, P2, and P4 are shown in Fig. $6 \mathrm{~b}$. P1 $\left(\lambda_{\max }=540 \mathrm{~nm}\right), \mathrm{P} 2\left(\lambda_{\max }=\right.$
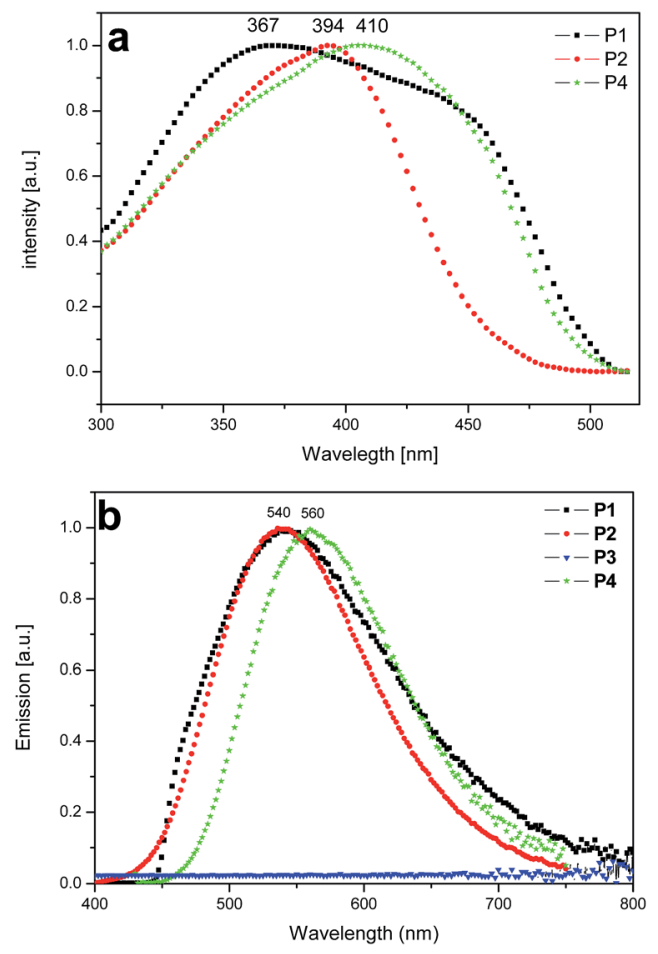

Fig. $6 \mathrm{PL}$ excitation spectra $\left(\lambda_{\text {det }}=550 \mathrm{~nm}\right)(\mathrm{a})$ and PL spectra $\left(\lambda_{\text {exc }}=\right.$ $400 \mathrm{~nm}$ ) (b) of P1 (black square), P2 (red circle), P3 (blue triangle), P4 (green star).
$540 \mathrm{~nm})$ and $\mathrm{P} 4\left(\lambda_{\max }=560 \mathrm{~nm}\right)$ display a yellow photoluminescence; P3 is non-emissive. P1 and P2 show quite moderate photoluminescence quantum yields (PLQY) of 3.5 and $6.5 \%$, respectively, for an excitation at $340 \mathrm{~nm}$. In contrast, $\mathrm{P} 4$ is characterized by a remarkably intense PL with a PLQY of $25.3 \%$ (excitation at $400 \mathrm{~nm}$; see Fig. 5). For P3, the combination of a comparatively low BET surface area $\left(200 \mathrm{~m}^{2} \mathrm{~g}^{-1}\right)$ with the absence of photoluminescence points for the formation of structural defects during network formation.

The photoluminescence excitation (PLE) spectra of the polymer networks P1, P2 and P4 are depicted in Fig. 6a with PLE maxima at $\lambda_{\max }=367 \mathrm{~nm}$ (shoulder at $c a .450 \mathrm{~nm}$ ) for P1, $\lambda_{\max }=$ $394 \mathrm{~nm}$ for P2 and $\lambda_{\max }=410 \mathrm{~nm}$ (shoulder at $c a .450 \mathrm{~nm}$ ) for P4 for a detection wavelength at $550 \mathrm{~nm}$.

Finally, the microporous polymer network $\mathrm{P} 4$ with its electron-deficient triazine cores has been tested for its interaction with electron-rich analytes as aromatic amines (Fig. 7).

For PL quenching tests P4-pellets have been exposed to saturated vapors of the amine analytes at atmospheric pressure and room temperature.

For $p$-toluidine $41 \% \mathrm{PL}$ quenching is observed after exposure for $1 \mathrm{~min}$, and $73 \%$ PL quenching after $45 \mathrm{~min}$. For aniline as analyte $39 \%$ PL quenching is observed after 1 min exposure and $82 \%$ after $49 \mathrm{~min}$. It can be assumed that the remarkable quenching response results from donor-acceptor interactions between the electron-rich aromatic analytes and the electronpoor triazine-based MPN P4. These initial results demonstrate a remarkable potential for the solid state sensing of organic analytes. Further experiments will be, especially, focused on the selectivity issue and on the reversibility of the PL quenching.
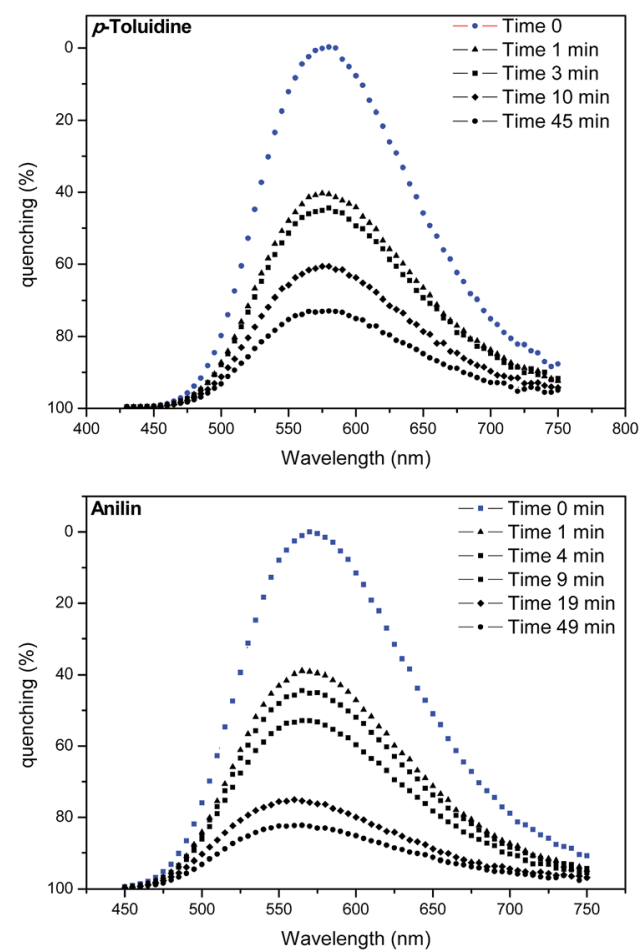

Fig. 7 PL quenching experiments with P4 pellets and $p$-toluidine or aniline as analytes. 


\section{Conclusions}

In conclusion, novel microporous polymer networks P1-P4 have been successfully synthesized via a reductive polyolefination protocol starting from tris( $\alpha, \alpha$-dichlorobenzyl)-substituted aromatic monomers with dicobalt octacarbonyl (preferred) or chromium(II)acetate as condensing agents. The most promising polymer network $\mathrm{P} 4$ that is composed of 1,3,5-triazine cores as linkers between aggregation induced emission (AIE)-active tetraphenylethylene units, reveals an intense yellow photoluminescence peaking at $560 \mathrm{~nm}$ with a remarkably high PLQY of $25.3 \%$ in line with a moderately high BET surface area of 475 $\mathrm{m}^{2} \mathrm{~g}^{-1}$. This combination of microporosity and high solid state PLQY (based on the AIE effect) clearly qualifies P4 with its electron deficient 1,3,5-triazine cores as an attractive target for further investigations, as its interaction with electron rich guests. Notably, first, orienting PL quenching experiments have demonstrated a distinct response to aromatic amine vapors (for aniline and $p$-toluidine).

\section{Acknowledgements}

The authors would like to thank P. Becker, J.-C. Gasse, and Dr. D. Lützenkirchen-Hecht for the powder XRD analysis.

\section{Notes and references}

1 A. Thomas, Angew. Chem., Int. Ed., 2010, 49, 8328-8344.

2 D. Wu, F. Xu, B. Sun, R. Fu, H. He and K. Matyjaszewski, Chem. Rev., 2012, 112, 3959-4015.

3 R. Dawson, A. I. Cooper and D. J. Adams, Prog. Polym. Sci., 2012, 37, 530-563.

4 N. B. McKeown and P. M. Budd, Chem. Soc. Rev., 2006, 35, 675-683.

5 T. Ben, H. Ren, S. Ma, D. Cao, J. Lan, X. Jing, W. Wang, J. Xu, F. Deng, J. M. Simmons, S. Qiu and G. Zhu, Angew. Chem., Int. Ed., 2009, 48, 9457-9460.

6 K. D. S. Kundu, J. Schmidt, C. Bleschke, A. Thomas and S. Blechert, Angew. Chem., Int. Ed., 2012, 51, 5456-5459.

7 R. R. Morris and P. S. Wheatley, Angew. Chem., Int. Ed., 2008, 47, 4966-4981.

$8 \mathrm{~J}$. Weber, M. Antonietti and A. Thomas, Macromolecules, 2007, 40, 1299-1304.

9 M. Rose, N. Klein, I. Senkovska, C. Schrage, P. Wollmann, W. Böhlmann, B. Böhringer, S. Fichtner and S. Kaskel, J. Mater. Chem., 2011, 21, 711-716.

10 K. T. Jackson, M. G. Rabbani, T. E. Reich and H. M. ElKaderi, Polym. Chem., 2011, 2, 2775-2777.

11 M. G. Schwab, B. Fassbender, H. W. Spiess, A. Thomas, X. Feng and K. Müllen, J. Am. Chem. Soc., 2009, 131, 72167217.

12 S. Ren, M. J. Bojdys, R. Dawson, A. Laybourn, Y. Z. Khimyak, D. J. Adams and A. I. Cooper, Adv. Mater., 2012, 24, 23572361.

13 Z. Wang, B. Zhang, H. Yu, L. Sun, C. Jiao and W. Liu, Chem. Commun., 2010, 46, 7730-7732.
14 Y. Kou, Y. Xu, Z. Guo and D. Jiang, Angew. Chem., Int. Ed., 2011, 50, 8753-8875.

15 Y. Luo, B. Li, L. Liang and B. Tan, Chem. Commun., 2011, 47, 7704-7706.

16 E. Preis, C. Widling, G. Brunklaus, J. Schmidt, A. Thomas and U. Scherf, ACS Macro Lett., 2013, 2, 380-383.

17 R. S. Sprick, A. Thomas and U. Scherf, Polym. Chem., 2010, 1, 283-285.

18 E. Preis, C. Widling, U. Scherf, S. Patil, G. Brunklaus, J. Schmidt and A. Thomas, Polym. Chem., 2011, 2, 2186-2189.

19 H.-H. Hörhold, M. Helbig, D. Raabe, J. Opfermann, U. Scherf, R. Stockmann and D. Weiß, Z. Chem., 1987, 27, 126-137.

20 Y. Hong, J. W. Y. Lam and B. Z. Tang, Chem. Soc. Rev., 2011, 40, 5361-5388.

21 A. Qin, J. W. Y. Lam and B. Z. Tang, Prog.Polym. Sci., 2012, 37, 182-209.

22 J. Zhou, Z. Chang, Y. Jiang, B. He, M. Du, P. Lu, Y. Hong, H. S. Kwok, A. Qin, H. Qiu, Z. Zhao and B. Z. Tang, Chem. Commun., 2013, 49, 2491-2493.

23 J. Liu, W. Z. Yuan, Y. Hu, B. Liu and B. Z. Tang, Adv. Funct. Mater., 2012, 22, 771-779.

24 W. Z. Yuan, P. Lu, S. Chen, J. W. Lam, Z. Wang, Y. Liu, H. S. Kwok, Y. Ma and B. Z. Tang, Adv. Mater., 2010, 22, 2159-2163.

25 Y. Xu, L. Chen, Z. Guo, A. Nagai and D. Jiang, J. Am. Chem. Soc., 2011, 133, 17622-17625.

26 A. Patra and U. Scherf, Chem.-Eur. J., 2012, 18, 10074-10080.

27 H. Reisch, U. Wiesler, U. Scherf and N. Tuytuylkov, Macromolecules, 1996, 29, 8204-8210.

28 E. Preis and U. Scherf, Macromol. Rapid Commun., 2006, 27, 1105-1109.

29 H.-H. Hörhold, J. Gottschaldt and J. Opfermann, J. Prakt. Chem., 1977, 319, 611-621.

30 C. Y. K. Chan, Z. Zhao, J. W. Y. Lam, J. Liu, S. Chen, P. Lu, F. Mahtab, X. Chen, H. H. Y. Sung, H. S. Kwok, Y. Ma, I. D. Williams, K. S. Wong and B. Z. Tang, Adv. Funct. Mater., 2012, 22, 378-389.

31 H.-H. Hörhold and D. Raabe, Acta Polym., 1979, 30, 86-92.

32 S. Kotha, D. Kashinath, K. Lahiri and R. B. Sunoj, Eur. J. Org. Chem., 2004, 4003-4013.

33 G. H. Miller, US Pat., 3775 380, 1973.

34 E. Ott, Ber. Dtsch. Chem. Ges. B, 1914, 52, 656-665.

35 C. Grundmann and E. Kober, J. Org. Chem., 1956, 21, 13921394.

36 C. L. Schmidt and M. Jansen, Eur. J. Inorg. Chem., 2012, 34, 5649-5657.

37 S.-H. Li, H.-P. Huang, S.-Y. Yu and X.-P. Li, Chin. J. Chem., 2006, 24, 1225-1229.

38 R. J. Milligan, C. B. Delano and T. J. Aponyi, J. Macromol. Sci., Chem., 1976, 10, 1467-1483.

39 S. Fischer, A. Schimanowitz, R. Dawson, I. Senkovska, S. Kaskel and A. Thomas, J. Mater. Chem. A, 2014, 2, 11825-11829.

$40 \mathrm{~J}$. Weber, M. Antonietti and A. Thomas, Macromolecules, 2008, 41, 2880-2885. 